\title{
A Non-linear Circle-preserving Subdivision Scheme
}

\author{
Pavel Chalmovianský \\ Johann Radon Institute of Computational and Applied Mathematics, \\ Austrian Academy of Science, Linz, Austria \\ pavel.chalmoviansky@oeaw.ac.at \\ Bert Jüttler \\ Institute of Applied Geometry, Johannes Kepler University, Linz, Austria \\ bert. juettler@jku. at
}

October 30, 2006

\begin{abstract}
We describe a new method for constructing a sequence of refined polygons, which starts with a sequence of points and associated normals. The newly generated points are sampled from circles which approximate adjacent points and the corresponding normals. By iterating the refinement procedure, we get a limit curve interpolating the data. We show that the limit curve is $G^{1}$, and that it reproduces circles. The method is invariant with respect to group of Euclidean similarities (including rigid transformations and scaling). We also discuss an experimental setup for a $G^{2}$ construction and various possible extensions of the method.
\end{abstract}

Keywords: subdivision techniques, fitting of algebraic curves

AMS Subject Classification: 65D17, 68U05, 53A04

\section{Introduction}

During the last years, iterative techniques for generating curve and surfaces have attracted a lot of attention, and they are now frequently being used in Computer Graphics and related areas. The classical linear schemes produce affinely invariant classes of curves and surfaces $[2,13]$. They are often obtained by generalizing the subdivision algorithms for certain spline functions, including trigonometric ones [8]. These techniques also include Hermite subdivision schemes [7], dealing with points with associated derivatives, see [3] and the references cited therein. Recently, several non-linear schemes have emerged, but these schemes - which seem to be promising - are much harder to analyze $[1,4,12]$.

On the other hand, various circle-based and circle-preserving techniques for generating curves are available $[5,9,10,11]$. Due to its technical importance, the reproduction of circular shapes is a desirable feature of constructions for planar curves.

We present a novel non-linear subdivision technique, which is able to reproduce circles. Starting with a sequence of points and associated normals, we compute a circle fit to any two neighboring points. Then, the new point is picked from this circle. It is shown that the circle is unique, and that the scheme produces $G^{1}$ limit curves for certain reasonable classes of input configurations. 
The construction can be modified in several ways, including different weights in the objective function used for the fitting, change of the fitted curve, and different strategies of choosing the new point. In particular, we propose a scheme which has experimentally been demonstrated to generate $G^{2}$ curves.

The paper is organized as follows. In section 2 we introduce briefly the notation and several necessary notions. Section 3 analyzes the basic step repeated during the refinement - the fitting of the circle to point and normal data. In section 4 , we recall the setup for subdivision of curves and adapt it to our construction. Section 5 provides the proof of several facts about the limit curve produced. The last section concludes the paper with several remarks concerning possible variations and improvements of the method.

\section{Preliminaries}

Let $P=\left\{\mathbf{p}_{i}\right\}_{i=0}^{n}$ be a sequence of points, where $\mathbf{p}_{i} \in \mathbb{R}^{2}$ for $i=0, \ldots, n$ and $V=\left\{\overrightarrow{\mathbf{n}}_{i}\right\}_{i=0}^{n}$ be a sequence of associated unit vectors, where $\overrightarrow{\mathbf{n}}_{i}=\left(\cos \varphi_{i}, \sin \varphi_{i}\right)^{\top} \in \mathbb{S}^{1}$ with $\varphi_{i} \in \mathbb{R}$ for $i=0, \ldots, n .^{1}$

In the method described below, the fundamental step of the construction consists in fitting a circle to a pair of neighboring points and associated normals. The newly generated points are then picked from it. The normal is determined as an appropriately oriented normal of the segment formed by those neighboring points. In each step, the total number of points is (roughly) doubled.

For an arbitrary vector $\overrightarrow{\mathbf{n}}=\left(n_{x}, n_{y}\right)^{\top}$, let $\overrightarrow{\mathbf{n}}^{\perp}=\left(-n_{y}, n_{x}\right)^{\top}$ be the vector obtained by a rotation of $\frac{\pi}{2}$.

\section{The Fundamental Construction Step}

Consider two points $\mathbf{a}, \mathbf{b} \in \mathbb{R}^{2}, \mathbf{a} \neq \mathbf{b}$ with associated normals $\overrightarrow{\mathbf{n}}_{\mathbf{a}}, \overrightarrow{\mathbf{n}}_{\mathbf{b}} \in \mathbb{S}^{1}$. We assume that $\|\mathbf{a}-\mathbf{b}\|=2 r$, where $r>0$. We construct a circle

$$
f(x, y)=0
$$

where

$$
f(x, y)=a\left(x^{2}+y^{2}\right)+b x+c y+d, \quad a, b, c, d \in \mathbb{R},
$$

which minimizes the objective function

$$
F(a, b, c, d)=f(\mathbf{a})^{2}+f(\mathbf{b})^{2}+\left\|\nabla f(\mathbf{a})-\overrightarrow{\mathbf{n}}_{\mathbf{a}}\right\|^{2}+\left\|\nabla f(\mathbf{b})-\overrightarrow{\mathbf{n}}_{\mathbf{b}}\right\|^{2} .
$$

Hence, the task is to solve

$$
\min _{a, b, c, d \in \mathbb{R}} F(a, b, c, d) .
$$

Clearly, the solution of (4) is invariant with respect to Euclidean transformations. Therefore, we suppose the following choice of the coordinate system (see Figure 1)

$$
\mathbf{a}=(-r, 0)^{\top}, \quad \mathbf{b}=(r, 0)^{\top}
$$

\footnotetext{
${ }^{1}$ For neighboring normals, we always take the closest representatives of their angles modulo $2 \pi$.
} 


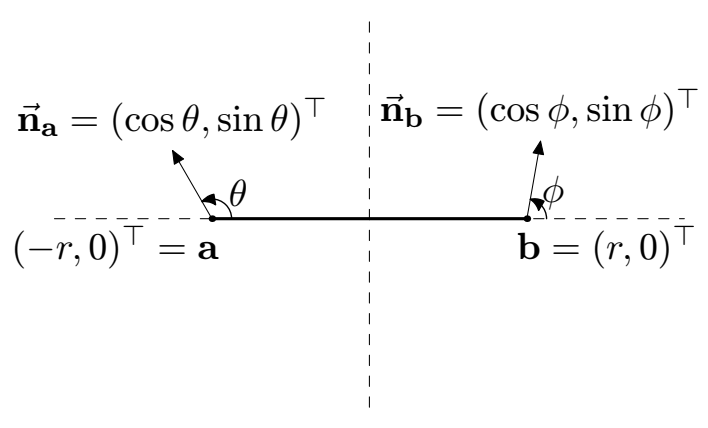

Figure 1: Local coordinates for analyzing the circle fit (Lemma 1).

and

$$
\overrightarrow{\mathbf{n}}_{\mathbf{a}}=(\cos \theta, \sin \theta)^{\top}, \quad \overrightarrow{\mathbf{n}}_{\mathbf{b}}=(\cos \phi, \sin \phi)^{\top}
$$

for $\theta, \phi \in \mathbb{R}$.

Note that - using a Euclidean transformation (rigid planar motion) - any two nonidentical points with associated normals can be mapped to this situation.

Let

$$
\alpha=\frac{\theta+\phi}{2} \text { and } \beta=\frac{\theta-\phi}{2} .
$$

The angles in (7) will be often referred to as functions

$$
\alpha\left(\mathbf{a}, \mathbf{b}, \overrightarrow{\mathbf{n}}_{\mathbf{a}}, \overrightarrow{\mathbf{n}}_{\mathbf{b}}\right) \text { and } \beta\left(\mathbf{a}, \mathbf{b}, \overrightarrow{\mathbf{n}}_{\mathbf{a}}, \overrightarrow{\mathbf{n}}_{\mathbf{b}}\right)
$$

of the given data. The following lemma shows that the circle fit always exists, except for pathological cases.

\section{Lemma 1}

(i) The problem (4) has a unique solution.

(ii) Let

$$
Z(f)=\left\{\mathbf{x} \in \mathbb{R}^{2}: f(\mathbf{x})=0\right\}
$$

be the zero set of the solution of (4). The set (9) is a real conic section unless $\theta=$ $-\phi=\frac{\pi}{2}+k \pi$ for $k \in \mathbb{Z}$. Moreover, if $\theta=\phi+2 k \pi$ or $\theta=-\phi+2 k \pi$ it is a double line. Otherwise, it is a circle.

Proof: The minimization of the function (3) leads to the $4 \times 4$ linear (inhomogeneous) system given by the Jacobi matrix

$$
\frac{\partial F(a, b, c, d)}{\partial(a, b, c, d)}=\overrightarrow{\mathbf{0}}
$$

One can easily check that the coefficient matrix has a non-zero determinant.

Now consider the set (9). Solving the system (10), we get

$$
a=\frac{\cos \theta-\cos \phi}{4 r}, \quad b=\frac{\cos \theta+\cos \phi}{2\left(r^{2}+1\right)}, \quad c=\frac{\sin \theta+\sin \phi}{2}, \quad d=-\frac{(\cos \theta-\cos \phi)}{4 r} .
$$

The coefficient $a$ vanishes iff

$$
\cos \theta-\cos \phi=0
$$


This condition is equivalent to

$$
\theta=\phi+2 k \pi
$$

or

$$
\theta=-\phi+2 k \pi \text { for } k \in \mathbb{Z}
$$

Clearly, the coefficient $d$ vanishes as well, if (12) holds. In the first case (13), either the coefficient $b \neq 0$ or $c \neq 0$, depending on the angles $\alpha$ and $\beta$. Therefore, the solution is a line

$$
b x+c y=0 .
$$

In the second case (14), we get the non-trivial solution unless the angles $\theta=\frac{\pi}{2}+k \pi$ and $\phi=-\frac{\pi}{2}+k \pi$, since then at least the coefficient $b \neq 0$.

If $a \neq 0$, the solution is a circle. The radius of the circle is equal to

$$
r_{f}=\sqrt{D_{f}} \quad \text { with } \quad D_{f}=\frac{b^{2}+c^{2}-4 a d}{4 a^{2}} .
$$

Consequently, there exists a real point on the circle if and only if

$$
D_{f} \geq 0 \text {. }
$$

The case $D_{f}=0$ represents a singular real solution - a point.

Substituting the solution (11) into the numerator of (17) and using trigonometric identities, we get an expression discriminating the existence of a real solution

$$
\sin ^{2} \alpha+\frac{1}{\left(r^{2}+1\right)^{2}} \cos ^{2} \alpha \cos ^{2} \beta \geq 0
$$

Clearly, it is always non-negative. Moreover, the strict inequality condition holds iff $\theta \neq \frac{\pi}{2}+k \pi$ or $\phi \neq-\frac{\pi}{2}+k \pi$ for any $k \in \mathbb{Z}$. Thus, we get a real circle in the generic case.

Assuming that $Z(f)$ is a circle, the newly generated point is taken from

$$
l \cap Z(f),
$$

where the line $l$ is the bisector of the points $\mathbf{a}$ and $\mathbf{b}$

$$
l: \mathbf{x}=\frac{1}{2} \mathbf{a}+\frac{1}{2} \mathbf{b}+t(\mathbf{b}-\mathbf{a})^{\perp}, \quad t \in \mathbb{R} .
$$

Using the choice of coordinates from Figure 1, it is the $y$-axis (see also Figure 2). After a short calculation, the new point turns out to be one of the two possible solutions

$$
\mathbf{q}^{\prime}=\left(0,-r \frac{\cos \beta+1}{\sin \beta}\right)^{\top} \text { and } \mathbf{q}=\left(0,-r \frac{\cos \beta-1}{\sin \beta}\right)^{\top} .
$$

Since the distance of each of the possible new points to the points $\mathbf{a}, \mathbf{b}$ is the same (due to the choice of line $l$ ), we get

$$
\left\|\mathbf{q}^{\prime}-\mathbf{a}\right\|^{2}=\left\|\mathbf{q}^{\prime}-\mathbf{b}\right\|^{2}=\frac{r^{2}}{\sin ^{2} \frac{\beta}{2}}
$$




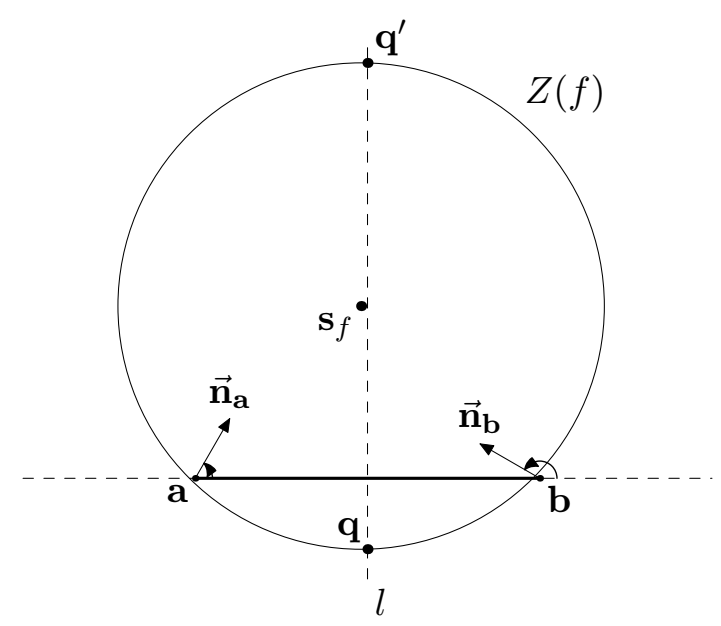

Figure 2: A new point generated from the fitted circle $Z(f)$ and the line $l$.

and

$$
\|\mathbf{q}-\mathbf{a}\|^{2}=\|\mathbf{q}-\mathbf{b}\|^{2}=\frac{r^{2}}{\cos ^{2} \frac{\beta}{2}} .
$$

We expect the new point to be in the vicinity of the existing ones and the corresponding angle $\beta$ is expected to be small for neighboring points. Consequently, we choose the point $\mathbf{q}$ as the one with the smaller distance.

If $Z(f)=\emptyset$ or the intersection of $l$ and $Z(f)$ (see (13) and (14)) contains more than finite number of points, we set

$$
\mathbf{q}=\frac{1}{2} \mathbf{a}+\frac{1}{2} \mathbf{b}
$$

Next, we need to determine an appropriate normal $\overrightarrow{\mathbf{n}}_{\mathbf{q}}$ associated with the new point $\mathbf{q}$. We assume that the normals $\overrightarrow{\mathbf{n}}_{\mathbf{a}}$ and $\overrightarrow{\mathbf{n}}_{\mathbf{b}}$ point to the same halfplane with respect to the line $\mathbf{a b}$. We take the normal of that line pointing in the same halfplane. This choice is invariant under all Euclidean transformations ${ }^{2}$.

Note that the normal can be calculated as an appropriately oriented normal of the circle passing through the points $\mathbf{a}, \mathbf{b}, \mathbf{q}$, when they are non-collinear due to (23). Hence, the construction reproduces circles.

Summarizing, we have the following

\section{Algorithm 2 (New Point and Normal)}

Given two points $\mathbf{a}, \mathbf{b}$ and two associated (unit) normals $\overrightarrow{\mathbf{n}}_{\mathbf{a}}, \overrightarrow{\mathbf{n}}_{\mathbf{b}}$.

If $\overrightarrow{\mathbf{n}}_{\mathbf{a}} \cdot \overrightarrow{\mathbf{n}}_{\mathbf{b}}>-1$, compute the new point from

$$
\mathbf{q}=\frac{1}{2} \mathbf{a}+\frac{1}{2} \mathbf{b}-\tan \frac{\beta}{2}(\mathbf{b}-\mathbf{a})^{\perp},
$$

where $\beta$ is given by (7). More precisely,

$$
\tan \frac{\beta}{2}=\operatorname{sgn}\left(\overrightarrow{\mathbf{n}}_{\mathbf{a}} \wedge \overrightarrow{\mathbf{n}}_{\mathbf{b}}\right)\left(\frac{1-c_{\beta}}{1+c_{\beta}}\right)^{\frac{1}{2}} \quad \text { with } \quad c_{\beta}=\left(\frac{1+\overrightarrow{\mathbf{n}}_{\mathbf{a}} \cdot \overrightarrow{\mathbf{n}}_{\mathbf{b}}}{2}\right)^{\frac{1}{2}},
$$

\footnotetext{
${ }^{2}$ Another choice would be to use simply the normalized vector $(\mathbf{b}-\mathbf{a})^{\perp}$. This works for all configurations of data, but it is not invariant under reflections.
} 
where $\overrightarrow{\mathbf{x}} \wedge \overrightarrow{\mathbf{y}}=x_{1} y_{2}-x_{2} y_{1}$. The corresponding normal is given as

$$
\overrightarrow{\mathbf{n}}_{\mathbf{q}}= \pm \frac{(\mathbf{b}-\mathbf{a})^{\top}}{\| \mathbf{b}-\mathbf{a}) \|}
$$

such that $\overrightarrow{\mathbf{n}}_{\mathbf{q}} \cdot \overrightarrow{\mathbf{n}}_{\mathbf{a}}>0$ and $\overrightarrow{\mathbf{n}}_{\mathbf{q}} \cdot \overrightarrow{\mathbf{n}}_{\mathbf{b}}>0$. We denote these functions as $\mathbf{q}\left(\mathbf{a}, \mathbf{b}, \overrightarrow{\mathbf{n}}_{\mathbf{a}}, \overrightarrow{\mathbf{n}}_{\mathbf{b}}\right)$ and $\overrightarrow{\mathbf{n}}\left(\mathbf{a}, \mathbf{b}, \overrightarrow{\mathbf{n}}_{\mathbf{a}}, \overrightarrow{\mathbf{n}}_{\mathbf{b}}\right)$.

Lemma 3 The choice of the new point and the new normal in Algorithm 2 is invariant under the group of Euclidean similarities (including rigid body transformations and scaling) of the plane.

Proof: The proof of invariance is obvious from (25) and (27).

An example is shown in Figure 3. Starting with the sequence of points and normals shown (i), it shows eight iterations of the refinement. Figure 4 provides a closer look at the behavior of the generated normals after the eighth iteration.

\section{Algebraic Subdivision of a Curve}

After introducing the notation and basic algorithm to refine a polygon, we will now prove some properties of the generated sequence of points. Let

$$
P^{0}=\left\{\mathbf{p}_{i}^{0}\right\}_{i=0}^{n_{0}} \quad \text { with } \quad \mathbf{p}_{i}^{0} \in \mathbb{R}^{2}
$$

such that

$$
\mathbf{p}_{i}^{0} \neq \mathbf{p}_{i+1}^{0} \quad \text { for } \quad i=0, \ldots, n_{0}-1
$$

and

$$
V^{0}=\left\{\overrightarrow{\mathbf{n}}_{i}^{0}\right\}_{i=0}^{n_{0}} \quad \text { with } \quad \overrightarrow{\mathbf{n}}_{i}^{0} \in \mathbb{S}^{1}
$$

be the sequence of associated unit normals with the points in $P^{0}$ for $n_{0} \in \mathbb{Z}, n_{0}>0$. Using Algorithm 2, we obtain iteratively a sequence of sequences, for $j=0,1,2, \ldots$,

$$
P^{j}=\left\{\mathbf{p}_{i}^{j}\right\}_{i=0}^{n_{j}} \quad \text { with } \quad \mathbf{p}_{i}^{j} \in \mathbb{R}^{2}
$$

and

$$
V^{j}=\left\{\overrightarrow{\mathbf{n}}_{i}^{j}\right\}_{i=0}^{n_{j}} \quad \text { with } \quad \overrightarrow{\mathbf{n}}_{i}^{j} \in \mathbb{S}^{1}
$$

such that

$$
\begin{gathered}
\mathbf{p}_{2 k+1}^{j+1}=\mathbf{q}\left(\mathbf{p}_{k}^{j}, \mathbf{p}_{k+1}^{j}, \overrightarrow{\mathbf{n}}_{k}^{j}, \overrightarrow{\mathbf{n}}_{k+1}^{j}\right) \text { for } k=0, \ldots, n_{j}-1, \\
\mathbf{p}_{2 k}^{j+1}=\mathbf{p}_{k}^{j} \text { for } k=0, \ldots, n_{j},
\end{gathered}
$$

are the newly generated points and

$$
\begin{gathered}
\overrightarrow{\mathbf{n}}_{2 k+1}^{j+1}=\overrightarrow{\mathbf{n}}\left(\mathbf{p}_{k}^{j}, \mathbf{p}_{k+1}^{j}, \overrightarrow{\mathbf{n}}_{k}^{j}, \overrightarrow{\mathbf{n}}_{k+1}^{j}\right) \quad \text { for } \quad k=0, \ldots, n_{j}-1, \\
\overrightarrow{\mathbf{n}}_{2 k}^{j+1}=\overrightarrow{\mathbf{n}}_{k}^{j} \quad \text { for } \quad k=0, \ldots, n_{j}
\end{gathered}
$$

are the newly generated vectors associated with the corresponding points, where

$$
n_{j+1}=2 n_{j}
$$




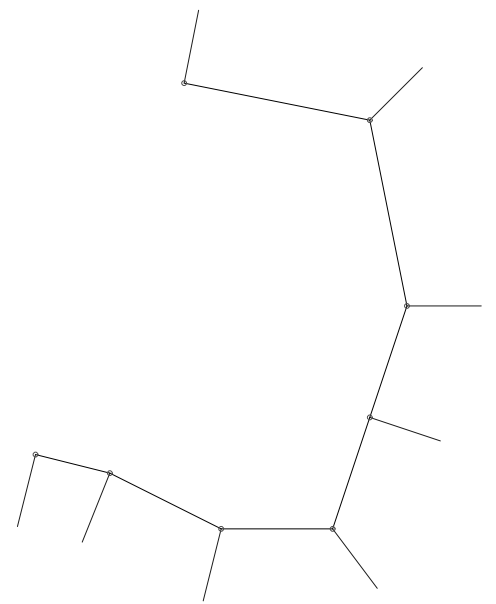

(i)

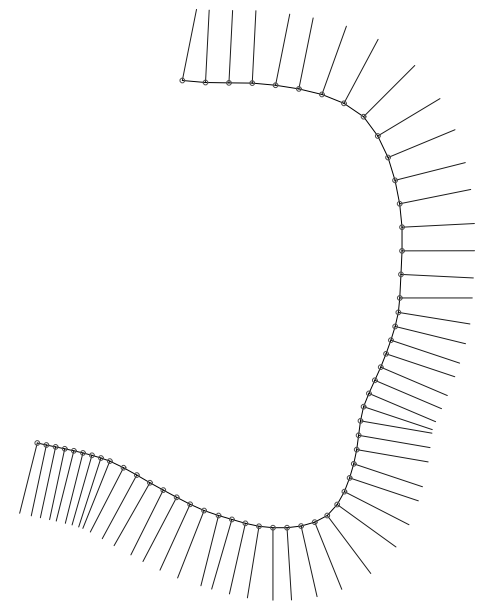

(iv)

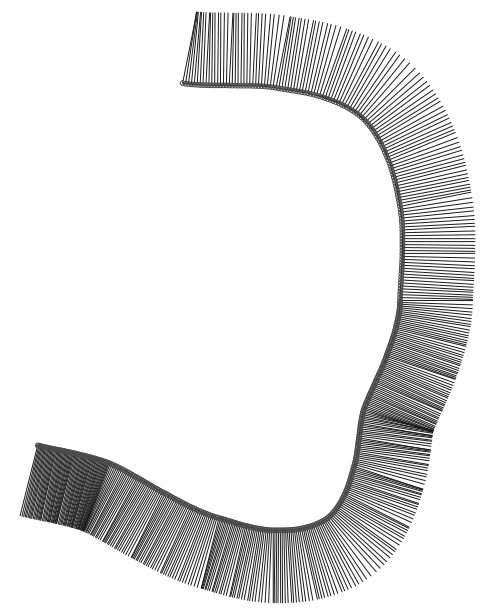

(vii)

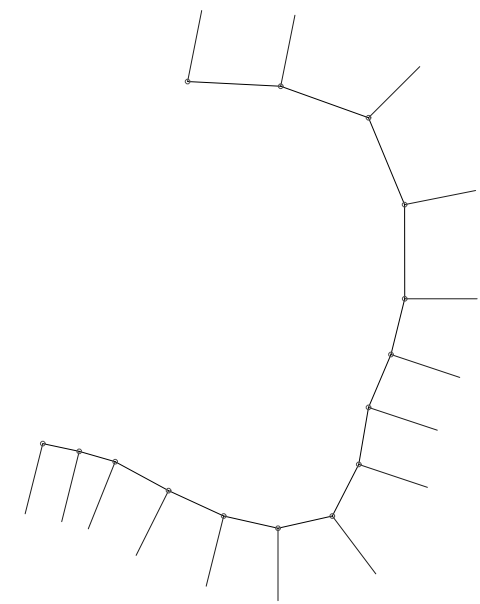

(ii)

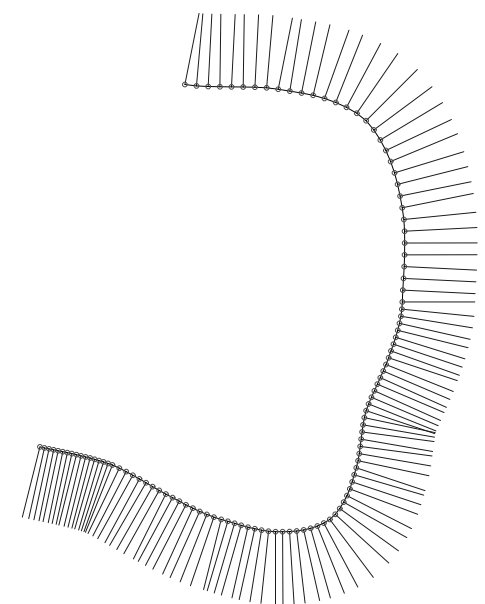

(v)

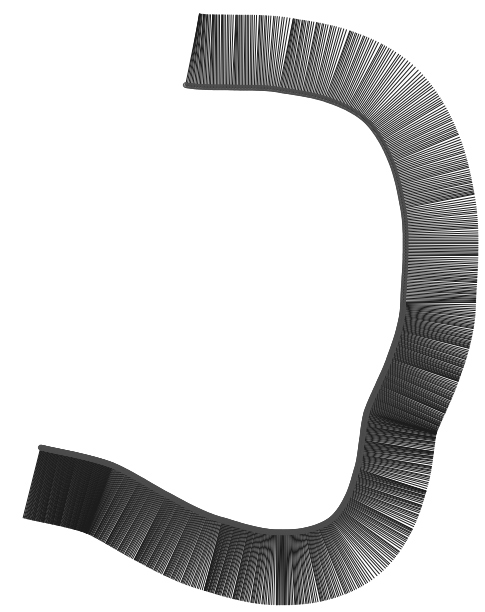

(viii)

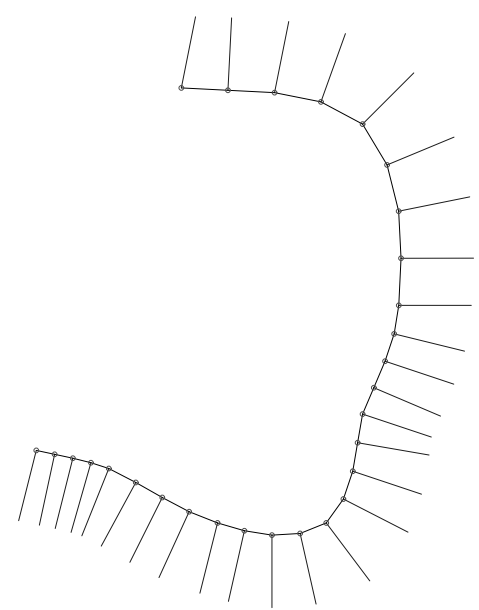

(iii)

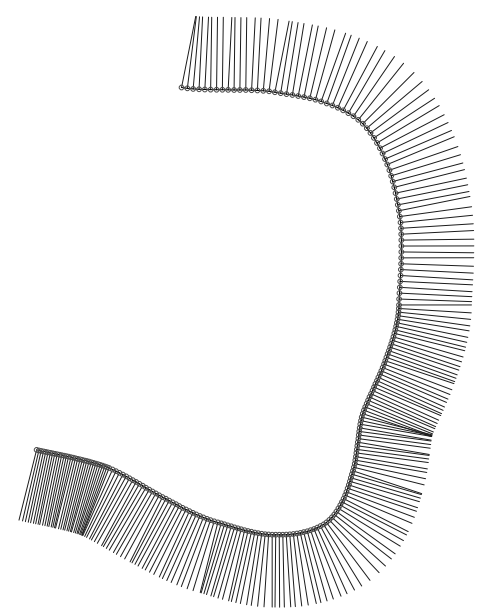

(vi)

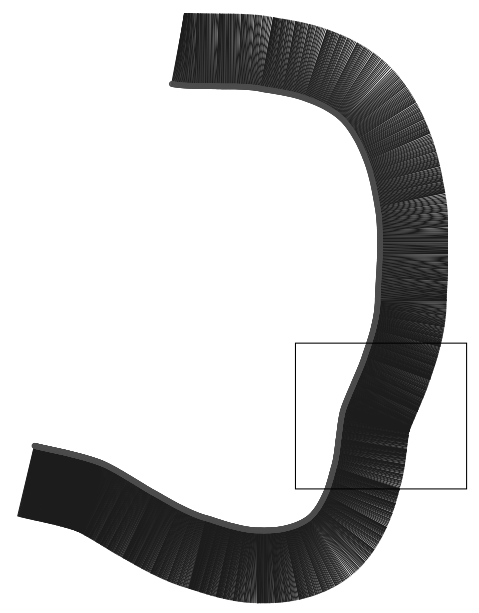

(ix)

Figure 3: Applying eight subdivision steps to the data in (i). The behavior of the framed part (ix) is shown in Figure 4. 


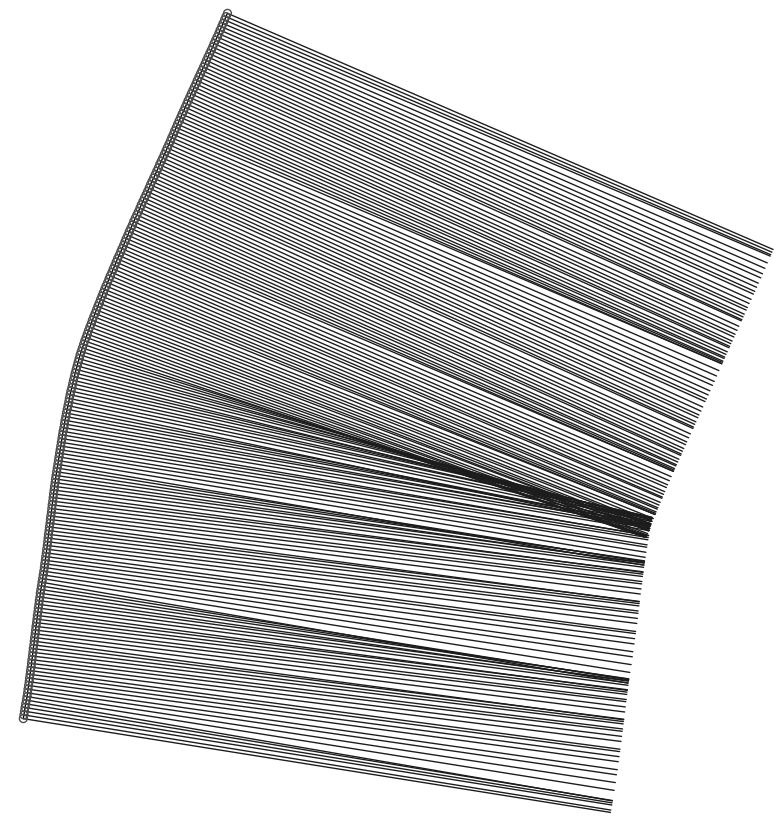

Figure 4: Zoom of the behavior of the normal for the Figure 3, (ix).

Associated with the sequences (31) and (32), we have also the sequences of local angles

$$
\left\{\theta_{i}^{j}\right\}_{i=0}^{n_{j}-1} \text { and } \quad\left\{\phi_{i}^{j}\right\}_{i=0}^{n_{j}-1}
$$

such that

$$
\overrightarrow{\mathbf{n}}_{i}^{j}=\left(\cos \theta_{i}^{j}, \sin \theta_{i}^{j}\right)^{\top}
$$

and

$$
\overrightarrow{\mathbf{n}}_{i+1}^{j}=\left(\cos \phi_{i}^{j}, \sin \phi_{i}^{j}\right)^{\top}
$$

in the local coordinate systems determined by the points $\mathbf{p}_{i}^{j}, \mathbf{p}_{i+1}^{j}$ (see Figure 1). Using (7), we also have an equivalent description

$$
\left\{\alpha_{i}^{j}\right\}_{i=0}^{n_{j}-1} \text { and } \quad\left\{\beta_{i}^{j}\right\}_{i=0}^{n_{j}-1}
$$

of the parameter angles for $j=0,1,2, \ldots$, where

$$
\alpha_{i}^{j}=\alpha\left(\mathbf{p}_{i}^{j}, \mathbf{p}_{i+1}^{j}, \overrightarrow{\mathbf{n}}_{i}^{j}, \overrightarrow{\mathbf{n}}_{i+1}^{j}\right)
$$

and

$$
\beta_{i}^{j}=\beta\left(\mathbf{p}_{i}^{j}, \mathbf{p}_{i+1}^{j}, \overrightarrow{\mathbf{n}}_{i}^{j}, \overrightarrow{\mathbf{n}}_{i+1}^{j}\right) .
$$

Note that the angles $\alpha_{i}^{j}$ and $\beta_{i}^{j}$ are also considered in the local coordinate systems defined by the neighboring points. Clearly, we may consider the sequences (31), (32) as sets $\bar{P}^{j}, \bar{V}^{j}$, and then

$$
\bar{P}^{j} \subset \bar{P}^{j+1} \quad \text { and } \quad \bar{V}^{j} \subset \bar{V}^{j+1}
$$

for $j=0,1,2, \ldots$. Let

$$
\bar{P}^{\infty}=\bigcup_{j=0}^{\infty} \bar{P}^{j} \quad \text { and } \quad \bar{V}^{\infty}=\bigcup_{j=0}^{\infty} \bar{V}^{j}
$$




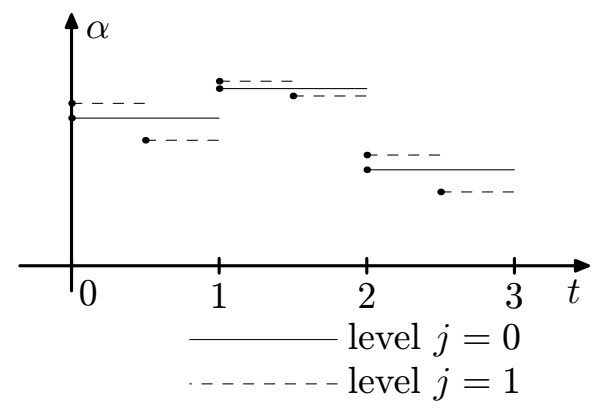

Figure 5: Change of the step function $\alpha$ during the refinement over the parameterization interval $\left[0, n_{0}\right)$ for $n_{0}=4$.

be the limit set of the generated points resp. vectors. We prove several properties of these sets.

Each of the sequences $P^{j}$ defines a polygon in $\mathbb{R}^{2}$. We denote it with poly $\left(P^{j}\right)$. It can be piecewise linearly parameterized. For $P^{0}$, we use

$$
\mathbf{p}^{0}(t)=\frac{t_{i+1}-t}{t_{i+1}-t_{i}} \mathbf{p}_{i}^{0}+\frac{t-t_{i}}{t_{i+1}-t_{i}} \mathbf{p}_{i+1}^{0}
$$

for $t \in\left[t_{i}, t_{i+1}\right)$, where $t_{i}=i$ for $i=0,1, \ldots, n_{0}$. Now, we can continue inductively with the parameterization of $\operatorname{poly}\left(P^{j}\right)$ as

$$
\mathbf{p}^{j}(t)=2^{j}\left(t_{i+1}^{j}-t\right) \mathbf{p}_{i}^{j}+2^{j}\left(t-t_{i}^{j}\right) \mathbf{p}_{i+1}^{j}
$$

for $t \in\left[t_{i}^{j}, t_{i+1}^{j}\right)$, where $t_{i}^{j}=\frac{i}{2^{j}}$ for $i=0,1, \ldots, n_{j}$. The parameters $t_{i}^{j}$ are called dyadic knots. Note that due to $(34)$

$$
\mathbf{p}^{j+1}\left(t_{2 i}^{j+1}\right)=\mathbf{p}^{j}\left(t_{i}^{j}\right)
$$

for $j=0,1, \ldots ; i=0,1, \ldots, n_{j}$. Similarly, we parameterize the corresponding vector function as

$$
\overrightarrow{\mathbf{n}}^{j}(t)=2^{j}\left(t_{i+1}^{j}-t\right) \overrightarrow{\mathbf{n}}_{i}^{j}+2^{j}\left(t-t_{i}^{j}\right) \overrightarrow{\mathbf{n}}_{i+1}^{j}
$$

for $t \in\left[t_{i}^{j}, t_{i+1}^{j}\right)$.

In addition, we can consider the sequence (41) as a sequence of piecewise constant functions, each defined on the interval $\left[0, n_{0}\right)$ with uniform binary refinement (see Figure 5).

Clearly, the values $\mathbf{p}\left(t_{i}^{j}\right)$ of the set $P^{\infty}$ and $\overrightarrow{\mathbf{n}}\left(t_{i}^{j}\right)$ of the set $V^{\infty}$ depend locally on the initial values. Hence, it suffices to consider a pair of neighboring points and normals to reveal the local properties of the generated sets.

In the sequel, we assume that

$$
\overrightarrow{\mathbf{n}}_{i}^{j} \cdot \overrightarrow{\mathbf{n}}_{i+1}^{j}>-1
$$

holds for $j=0,1, \ldots, i=0, \ldots, n_{j}-1$.

\section{Analysis of the Limit Curve}

Consider the sequence $P^{0}=\{\mathbf{a}, \mathbf{b}\}$ for $\mathbf{a} \neq \mathbf{b}$ as in (5) and $V^{0}=\left\{\overrightarrow{\mathbf{n}}_{\mathbf{a}}, \overrightarrow{\mathbf{n}}_{\mathbf{b}}\right\}$ as defined in (6). Hence, $n_{0}=1$ in this section. 
We will prove that the generated set $\bar{P}^{\infty}$ is a dense subset of $G^{1}$ curve and, moreover, the normals of that curve are those in $\bar{V}^{\infty}$ for the corresponding points.

The proof consists of the following steps.

1. First, we prove the technical Lemma 4, which preserves certain configurations of data during the subdivision and certain properties of sequences of local angles.

2. Second, we show that the polygons generated by our algorithm, provided the initial data are in certain configurations, form a Cauchy sequence in the space $C^{0}[0,1]$ with respect to the maximum norm.

3. We prove that the limit curve has finite length and it is locally injective.

4. As the last step, we show that the limit of that sequence of $C^{0}$ functions is $G^{1}$, by considering the existence of tangent in each generated point.

\subsection{Continuity of the limit curve}

The following technical lemma is a key tool for the proof of the properties of the limit sets (45).

Lemma 4 If

$$
\begin{array}{r}
\left|\alpha_{0}^{0}-\frac{\pi}{2}\right| \leq \frac{\pi}{4}, \\
\left|\beta_{0}^{0}\right| \leq \frac{\pi}{4}
\end{array}
$$

hold, then

$$
\begin{aligned}
\left(\begin{array}{c}
\alpha_{2 i}^{j+1}-\frac{\pi}{2} \\
\beta_{2 i}^{j+1}
\end{array}\right) & =\left(\begin{array}{cc}
\frac{1}{2} & 0 \\
\frac{1}{2} & \frac{1}{2}
\end{array}\right)\left(\begin{array}{c}
\alpha_{i}^{j}-\frac{\pi}{2} \\
\beta_{i}^{j}
\end{array}\right), \text { and } \\
\left(\begin{array}{c}
\alpha_{2 i+1}^{j+1}-\frac{\pi}{2} \\
\beta_{2 i+1}^{j+1}
\end{array}\right) & =\left(\begin{array}{cc}
\frac{1}{2} & 0 \\
-\frac{1}{2} & \frac{1}{2}
\end{array}\right)\left(\begin{array}{c}
\alpha_{i}^{j}-\frac{\pi}{2} \\
\beta_{i}^{j}
\end{array}\right)
\end{aligned}
$$

for $j=0,1, \ldots$ and $i=0,1, \ldots, n_{j}-1$. In particular,

$$
\begin{aligned}
\left|\alpha_{2 i}^{j+1}-\frac{\pi}{2}\right| & =\left|\alpha_{2 i+1}^{j+1}-\frac{\pi}{2}\right| \leq \frac{\pi}{4}, \\
\left|\beta_{2 i}^{j+1}\right| & \leq \frac{\pi}{4}, \text { and } \\
\left|\beta_{2 i+1}^{j+1}\right| & \leq \frac{\pi}{4} .
\end{aligned}
$$

Proof: We prove the statement by induction on $j$. For $j=0$, see Figure 6 , where the indices of $\alpha$ and $\beta$ were omitted in order to simplify the figure.

The new angles after a subdivision step in the local coordinates determined by points $\mathbf{a}, \mathbf{q}$ are $\theta_{0}^{1}=\alpha_{0}^{0}+\frac{\beta_{0}^{0}}{2}$ and $\phi_{0}^{1}=\frac{\pi}{2}-\frac{\beta_{0}^{0}}{2}$. Similarly, $\theta_{1}^{1}=\frac{\pi}{2}+\frac{\beta_{0}^{0}}{2}$ and $\phi_{1}^{1}=\alpha_{0}^{0}+\frac{\beta_{0}^{0}}{2}$ are the angles for the coordinate system determined by points $\mathbf{q}, \mathbf{b}$. After a short calculation, using (7), we get 


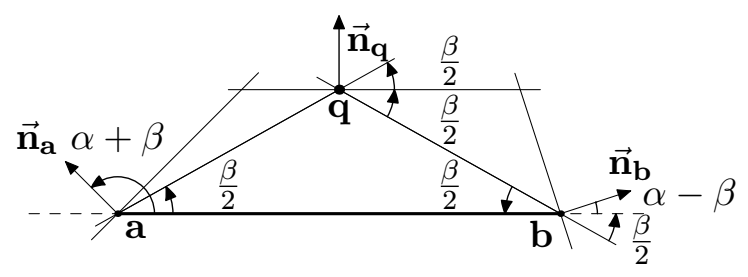

Figure 6: Angles in a subdivision step

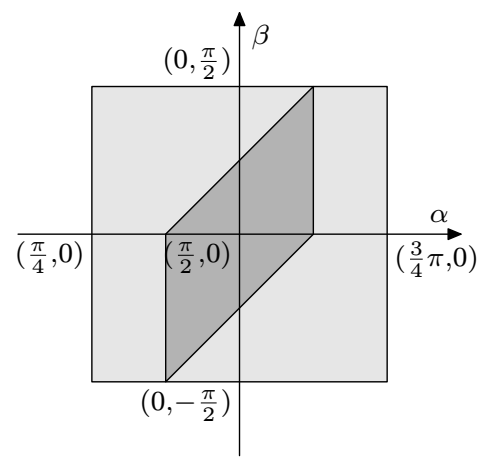

Transformation $A_{L}$

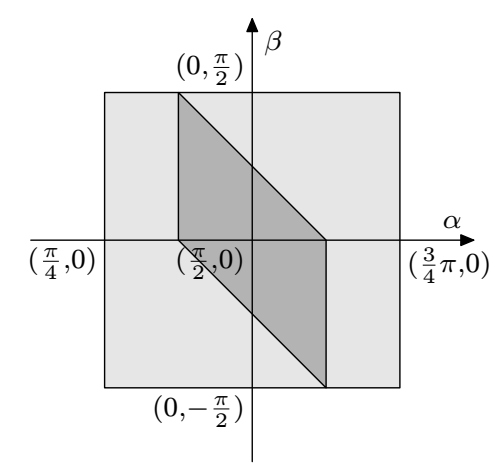

Transformation $A_{R}$

Figure 7: The angle transformations (53) and (54)

$$
\begin{aligned}
\alpha_{1}^{1}-\frac{\pi}{2} & =\alpha_{0}^{1}-\frac{\pi}{2}=\frac{1}{2}\left(\alpha_{0}^{0}-\frac{\pi}{2}\right) \\
\beta_{0}^{1} & =\frac{1}{2}\left(\alpha_{0}^{0}-\frac{\pi}{2}\right)+\frac{1}{2} \beta_{0}^{0} \\
\beta_{1}^{1} & =-\frac{1}{2}\left(\alpha_{0}^{0}-\frac{\pi}{2}\right)+\frac{1}{2} \beta_{0}^{0},
\end{aligned}
$$

which are the transformations (53) and (54). Clearly,

$$
\begin{aligned}
\left|\alpha_{i}^{1}-\frac{\pi}{2}\right| & \leq \frac{1}{2}\left|\alpha_{i}^{1}-\frac{\pi}{2}\right| \leq \frac{\pi}{4} \\
\left|\beta_{i}^{1}\right| & \leq \frac{1}{2} \cdot \frac{\pi}{4}+\frac{1}{2} \cdot \frac{\pi}{4}=\frac{\pi}{4}
\end{aligned}
$$

for $i \in\{0,1\}$. Hence (55), (56) and (57) hold.

Now, we can inductively proceed to finish the proof for $j \geq 1$.

We will denote the $2 \times 2$ matrix from (53) as $A_{L}$ and the matrix from (54) as $A_{R}$. The transformation of the angles can be visualized by Figure 7 . The light grey square area is the feasible domain of inequalities (51) and (52), the dark grey area is its transformation under the transformations given by (53) and (54). It is easy to see that both mappings are contractive. 
Lemma 5 If the conditions (51), (52) hold, the sequence of sequences (41) has the following properties: For $k=0,1, \ldots, j=0,1, \ldots, i=0, \ldots, n_{j}-1$

$$
\begin{aligned}
\left|\alpha_{i}^{j}-\frac{\pi}{2}\right| & =\frac{1}{2^{j}}\left|\alpha_{0}^{0}-\frac{\pi}{2}\right| \\
\left|\beta_{i}^{j}\right| & \leq \frac{\left|\beta_{0}^{0}\right|}{2^{j}}+\frac{j\left|\alpha_{0}^{0}-\frac{\pi}{2}\right|}{2^{j}} \\
\alpha_{i}^{j}-\frac{\pi}{2} & =\sum_{l=2^{k} i}^{2^{k}(i+1)-1}\left(\alpha_{l}^{j+k}-\frac{\pi}{2}\right), \text { and } \\
\beta_{i}^{j} & =\sum_{l=2^{k} i}^{2^{k}(i+1)-1} \beta_{l}^{j+k} .
\end{aligned}
$$

Proof: Using (58), (59) and (60), we get all statements of this lemma by induction.

Lemma 6 If the conditions (51) and (52) hold, the sequence of polygons

$$
\left\{\mathbf{p}^{j}(t)\right\}_{j=0}^{\infty}
$$

converges to a $C^{0}$ curve. We will refer to this limit as $\mathbf{p}(t)$.

Proof: Considering the parameterization (47) and using (23), we get

$$
\left\|\mathbf{p}^{j+1}(t)-\mathbf{p}^{j}(t)\right\| \leq r_{\max }^{j} \frac{1}{\cos \frac{\beta_{\max }^{j}}{2}}
$$

for sufficiently large $j$, where

$$
r_{\max }^{j}=\frac{1}{2} \max _{i=0, \ldots, n_{j}-1}\left\|\mathbf{p}_{i+1}^{j}-\mathbf{p}_{i}^{j}\right\|
$$

and

$$
\beta_{\max }^{j}=\max _{i=0, \ldots, n_{j}-1}\left|\beta_{i}^{j}\right|
$$

Using (23), we get

$$
r_{\max }^{j+1} \leq \frac{1}{2\left|\cos \frac{\beta_{\max }^{j}}{2}\right|} r_{\max }^{j} .
$$

Due to (64), $\beta_{\max }^{j} \rightarrow 0$ as $j \rightarrow \infty$. Hence, there is an constant $D, 0<D<1$ and an integer $j_{0} \in \mathbb{Z}_{+}$such that

$$
0<\frac{1}{2\left|\cos \frac{\beta_{\max }^{j}}{2}\right|}<D<1
$$

hold for all $j>j_{0}$. Summing up,

$$
\left\|\mathbf{p}^{j+1}(t)-\mathbf{p}^{j}(t)\right\| \leq 2 r_{\max }^{j_{0}} D^{j+1-j_{0}}
$$

for $j>j_{0}$ and therefore (67) is a Cauchy sequence of $C^{0}$ polygons. Therefore, it converges uniformly to a $C^{0}$ curve $\mathbf{p}(t)$. 


\subsection{Several properties of the limit curve}

Now we prove several properties of the limit curve $\mathbf{p}(t)$ from Lemma 6 . Let $\overrightarrow{\mathbf{i}}=\left\{i_{j}\right\}_{j=0}^{\infty}$ be a sequence of integers. Let

$$
D_{\infty}=\left\{\overrightarrow{\mathbf{i}}: i_{j} \in\left\{0, \ldots, n_{j}-1\right\} \quad \& \quad\left(i_{j+1}=2 i_{j} \quad \vee \quad i_{j+1}=2 i_{j}+1 \quad \text { for } \quad j \geq 0\right)\right\} .
$$

Further, let

$$
D_{n}=\left\{\left\{i_{j}\right\}_{i=0}^{n}: \overrightarrow{\mathbf{i}} \in D_{\infty}\right\}
$$

be the set of all finite sequences of length $n$, which can be extended to a sequence from $D_{\infty}$. Clearly, each sequence $\overrightarrow{\mathbf{i}} \in D_{\infty}$ induces a sequence of intervals $\left[t_{i_{j}}^{j}, t_{i_{j}+1}^{j}\right)$ with dyadic parameters as endpoints. An interval on the level $j+1$ is either the left or the right offspring of the interval on the level $j$ generated during subdivision. Similarly, we can also form a (finite or infinite) sequences of corresponding angles,

$$
\alpha_{\overrightarrow{\mathbf{i}}}=\left\{\alpha_{i_{j}}^{j}\right\}_{j=0}^{\infty} \quad \text { and } \quad \beta_{\overrightarrow{\mathbf{i}}}=\left\{\beta_{i_{j}}^{j}\right\}_{j=0}^{\infty} .
$$

For a sequence $\mathbf{y}=\left\{y_{i}\right\}_{i=0}^{\infty}$ and $c \in \mathbb{R}$, we use the abbreviations

$$
(\mathbf{y}, c)=\left\{y_{i}-c\right\}_{i=0}^{\infty} \quad \text { and } \quad S_{k}(\mathbf{y}, c)=\sum_{j=0}^{\infty}\left(y_{i}-c\right)^{k} .
$$

Further, for any sequence $\mathbf{x}=\left\{x_{i}\right\}_{i=0}^{\infty}$, let

$$
\mathbf{y} \circ \mathbf{x}=\left\{y_{i} x_{i}\right\}_{i=0}^{\infty} .
$$

The sequence of polygons generated during the subdivision has the following property.

Lemma 7 If (51) and (52) hold, then the lengths of the polygons $\mathbf{p}^{j}(t)$ converge to a finite limit as $j \rightarrow \infty$.

Proof: Let $l_{n}$ be the length of the polygon $\mathbf{p}^{n}(t)$. Starting with the situation on Figure 1, we have $l_{0}=2 \mathrm{r}$. Using (23) and induction, we get

$$
l_{n}=2 r \frac{1}{2^{n-1}} \sum_{\overrightarrow{\mathbf{i}} \in D_{n}} \prod_{j=0}^{n-1}\left(\cos \frac{1}{2} \beta_{i_{j}}^{j}\right)^{-1} .
$$

Due to $(23)$ and (64) the sequence $\left\{l_{n}\right\}_{n=0}^{\infty}$ is non-decreasing for all $n>\bar{n}$ for some $\bar{n} \in \mathbb{Z}_{+}$. We prove that it is also bounded.

Using the well-known Taylor expansion

$$
\cos x=\sum_{i=0}^{\infty}(-1)^{i} \frac{x^{2 i}}{(2 i) !}
$$

we get in a certain neighborhood of 0 the following estimate

$$
\cos x \geq 1-\frac{x^{2}}{2}
$$


According to (64), we can assume without loss of generality that all angles $\beta_{i_{j}}^{j}$ are sufficiently small for $j \geq 0$. If not, we can refine the data finitely many times and get the needed inequality for $\beta_{i_{j}}^{j}$ for some $j \geq j_{0}$ on the finitely many subintervals. Therefore

$$
\prod_{j=0}^{n-1}\left(\cos \frac{1}{2} \beta_{i_{j}}^{j}\right)^{-1} \leq \prod_{j=0}^{\infty}\left(\cos \frac{\beta_{i_{j}}^{j}}{2}\right)^{-1} \leq \prod_{j=0}^{\infty}\left(1-\frac{\left(\beta_{i_{j}}^{j}\right)^{2}}{8}\right)^{-1}
$$

for any $\overrightarrow{\mathbf{i}} \in D_{\infty}$ expanding the given finite one. The product on the right-hand side of (82) is convergent iff

$$
S_{2}\left(\beta_{\overrightarrow{\mathbf{i}}}, 0\right)=\sum_{j=0}^{\infty}\left(\beta_{i_{j}}^{j}\right)^{2}
$$

converges.

Clearly, the series $S_{2}\left(\alpha_{\overrightarrow{\mathbf{i}}}, \frac{\pi}{2}\right), S_{1}\left(\beta_{\overrightarrow{\mathbf{i}}}, 0\right)$ and $S_{1}\left(\alpha_{\overrightarrow{\mathbf{i}}}, \frac{\pi}{2}\right)$ are absolutely convergent due to (63) and (64). Hence, using (53) and (54) we get

$$
\begin{aligned}
S_{2}\left(\beta_{\overrightarrow{\mathbf{i}}}, 0\right) & =\left(\beta_{0}^{0}\right)^{2}+\frac{1}{4}\left(S_{2}\left(\beta_{\overrightarrow{\mathbf{i}}}, 0\right)+S_{2}\left(\alpha_{\overrightarrow{\mathbf{i}}}, \frac{\pi}{2}\right)\right) \\
& +\frac{1}{2} S_{1}\left(\left(\beta_{\overrightarrow{\mathbf{i}}}, 0\right) \circ\left(\alpha_{\overrightarrow{\mathbf{i}}}, \frac{\pi}{2}\right) \circ\left(\left\{(-1)^{\left(i_{j+1} \quad \bmod 2\right)}\right\}_{j=0}^{\infty}, 0\right), 0\right) .
\end{aligned}
$$

Solving (84) for $S_{2}\left(\beta_{\overrightarrow{\mathbf{i}}}, 0\right)$, we get the convergence of (83) and therefore (82) is also convergent. Using (64), it is also bounded.

Since $\left|D_{n}\right|=2^{n}$, we may conclude that $\left\{l_{n}\right\}_{n=0}^{\infty}$ is bounded. This completes the proof.

Lemma 8 The arc length of the polygon $\mathbf{p}^{j}(t)$ has a uniform lower bound with respect to the length of the domain interval.

Proof: Let $I=\left[t_{k}^{j}, t_{k+l}^{j}\right] \subset[0,1]$. Due to (23), we get

$$
\left\|\mathbf{p}_{i+1}^{j}-\mathbf{p}_{i}^{j}\right\| \geq r \frac{1}{2^{j-1}}
$$

for all $i \in\left\{0, \ldots, n_{j}-1\right\}$. Hence, the length of the polygon $\mathbf{p}_{k}^{j}, \ldots, \mathbf{p}_{k+l}^{j}$ can be estimated as

$$
l\left(\left.\mathbf{p}^{j}(t)\right|_{I}\right) \geq r \frac{1}{2^{j-1}} \cdot l=2 r|I| .
$$

where $2 r$ is the distance of the two initial points.

Let $I \subset[0,1]$ be an interval with dyadic endpoints. We will consider the turning angle of the generated normals over the interval $I$ at level $j$. Let

$$
\omega_{i, i+1}^{j}=-\left(\theta_{i}^{j}-\frac{\pi}{2}\right)+\left(\phi_{i}^{j}-\frac{\pi}{2}\right)=-2 \beta_{i}^{j},
$$

see Figure 8. Similarly, for $l>0$,

$$
\omega_{k, k+l}^{j}=\sum_{i=0}^{l-1} \omega_{k+i, k+i+1}^{j}=-2 \sum_{i=0}^{l-1} \beta_{k+i}^{j} .
$$




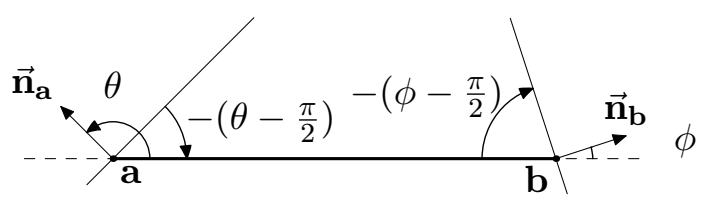

Figure 8: The definition of $\omega_{i, i+1}^{j}$ (corresponding indices are omitted).

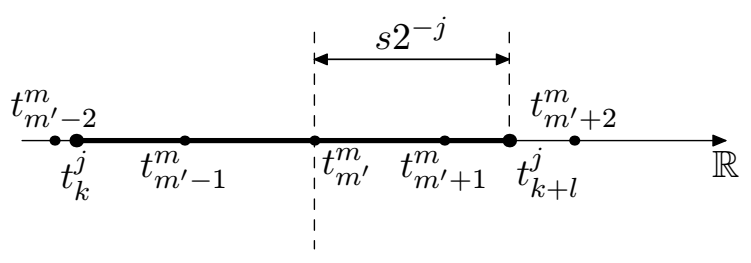

Figure 9: Estimation of the length of $I$ via the length of its maximum dyadic subinterval of level $m$.

Also, let $\omega_{k, k}^{j}=0$ for all feasible $j$ and $k$. Due to (66), we have for a polygon with given two endpoints after a subdivision step

$$
\omega_{k, k+l}^{j}=\omega_{2 k, 2 k+2 l}^{j+1} .
$$

On the other hand, let $\bar{\omega}_{k, l}^{j}$ be the turning angle of the polygon $\mathbf{p}_{k}^{j}, \ldots, \mathbf{p}_{l}^{j}$. Clearly,

$$
\bar{\omega}_{k, l}^{j}-\omega_{k, l}^{j}=\theta_{k}^{j}-\frac{\pi}{2}-\left(\phi_{l-1}^{j}-\frac{\pi}{2}\right)=\left(\alpha_{k}^{j}+\beta_{k}^{j}-\frac{\pi}{2}\right)-\left(\alpha_{l-1}^{j}-\beta_{l-1}^{j}-\frac{\pi}{2}\right) .
$$

Due to (63) and (64), the right side of the (90) converge to zero as $j \rightarrow \infty$ and therefore the turning angle of the normals converges to the turning angle of the curve $\mathbf{p}(t)$ for any given two endpoints.

We now bound the value of $\omega_{k, k+l}^{j}$ with respect to the length of the corresponding parameter interval $\left[t_{k}^{j}, t_{k+l}^{j}\right]$.

Let $m$ be the smallest non-negative integer such that for some $m^{\prime} \in\left\{0,1, \ldots, n_{m}-1\right\}$

$$
\left[t_{m^{\prime}}^{m}, t_{m^{\prime}+1}^{m}\right] \subset\left[t_{k}^{j}, t_{k+l}^{j}\right]
$$

Without loss of generality we suppose that $m^{\prime}$ is the biggest number satisfying (91). Since the number $m$ is the minimum, we can have at most one more dyadic point of of level $m$ in the interval $I$. Then the length of the interval can be estimated as

$$
2^{-m} \leq|I|=\frac{l}{2^{j}} \leq 2^{-m+2},
$$

(for the right inequality see also Figure 9). Further, let

$$
s=l-m^{\prime} 2^{j-m}=s_{0} 2^{j-m}+s_{1} 2^{j-(m+1)}+\cdots+s_{j-m}
$$

with $s_{i} \in\{0,1\}$ for $i=0, \ldots, j-m$ and $s_{0}=1$ (see Figure 9). Let

$$
S_{i}=s_{0} 2^{j-m}+s_{1} 2^{j-m-1}+\cdots+s_{i} 2^{j-m-i}
$$


be partial sums for $i \in\{0,1, \ldots, j-m\}$ and $S_{-1}=0$. Then

$$
\omega_{m^{\prime} 2^{j-m}, l}^{j}=\sum_{i=0}^{j-m} \omega_{m^{\prime} 2^{j-m}+S_{i-1}, m^{\prime} 2^{j-m}+S_{i}}^{j}
$$

Therefore,

$$
\begin{aligned}
\left|\omega_{m^{\prime} 2^{j-m}, l}^{j}\right| & \leq \sum_{i=0}^{j-m}\left|\omega_{m^{\prime} 2^{j-m}+S_{i-1}, m^{\prime} 2^{j-m}+S_{i}}^{j}\right| \leq \sum_{i=0}^{j-m} 2 s_{i}\left|\beta_{m^{\prime} 2^{i}+2^{-(j-(m+i))} S_{i-1}}^{m+i}\right| \\
& \leq 2 \sum_{i=0}^{j-m} \frac{\left|\beta_{0}^{0}\right|}{2^{m+i}}+\frac{(m+i)\left|\alpha_{0}^{0}-\frac{\pi}{2}\right|}{2^{m+i}} \leq \frac{C(m)}{2^{m}}
\end{aligned}
$$

where

$$
C(m)=4\left|\beta_{0}^{0}\right|+4(m+1)\left|\alpha_{0}^{0}-\frac{\pi}{2}\right| .
$$

Similarly, we can estimate

$$
\left|\omega_{k, m^{\prime} 2^{j-m}}^{j}\right| \leq \frac{C(m)}{2^{m}}
$$

Note that it may occur $t_{m^{\prime}-1}^{m}<t_{k}^{j}$ and therefore the $\left|\omega_{k, m^{\prime} 2^{j-m}}^{j}\right|$ angle of the left part might have even a smaller upper bound. Combining (96) and (98), we get

$$
\left|\omega_{k, k+l}^{j}\right| \leq \frac{2 C(m)}{2^{m}}
$$

Hence, we can prove the following Lemma.

Lemma 9 The turning angle of the generated normals (49) for $\mathbf{p}^{j}(t)$ is uniformly continuous with respect to the length of a parametric interval for all $j$.

Proof: Let $I \subset[0,1]$ be an interval with dyadic endpoints such that $|I|<2^{-M}$. Let $m$ be the minimum integer such that $2^{-m} \leq|I|$. Then

$$
M<m
$$

holds. Hence,

$$
\left|\omega_{k_{j}, l_{j}}^{j}\right| \leq \frac{2 C(M)}{2^{M}}
$$

where $I=\left[t_{k_{j}}^{j}, t_{l_{j}}^{j}\right]$ for $j>j(M)$. Since the right-hand side of (101) can be made arbitrarily small for $|I| \rightarrow 0$, we have the desired result.

Corollary 10 For every $\mathbf{p}_{i}^{j}, j=0,1, \ldots, i \in\left\{0, \ldots, n_{j}\right\}$, there is $h_{0} \in \mathbb{Z}_{+}, h_{0} \geq j$ and $\epsilon>0$ such that if $0<\left|t-t_{i}^{j}\right|<\epsilon$ then

$$
\left\|\mathbf{p}^{h}(t)-\mathbf{p}^{h}\left(t_{i}^{j}\right)\right\|>0
$$

holds for all $h>h_{0}$. 


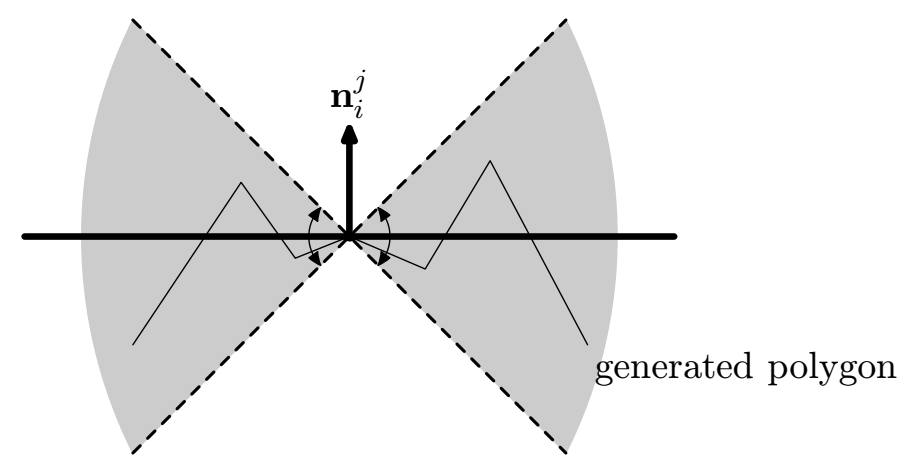

Figure 10: The local injectivity of the generated points.

Proof: First, we consider all dyadic points for parameters $t_{i^{\prime}}^{j^{\prime}},\left|t_{i^{\prime}}^{j^{\prime}}-t_{i}^{j}\right|<\epsilon, t_{i^{\prime}}^{j^{\prime}} \neq t_{i}^{j}$. Let $h_{0}$ be such that for all $h \geq h_{0}$

$$
\left|\alpha_{k}^{h}+\beta_{k}^{h}-\frac{\pi}{2}\right|<\epsilon \quad \text { and } \quad\left|\alpha_{l-1}^{h}-\beta_{l-1}^{h}-\frac{\pi}{2}\right|<\epsilon
$$

for some sufficiently small $\epsilon>0$ and feasible $k, l \in \mathbb{Z}_{+}$. Let $M$ be such that

$$
\frac{2 C(M)}{2^{M}}<\frac{\pi}{4}-2 \epsilon
$$

Then, for all $\left|t_{i^{\prime}}^{j^{\prime}}-t_{i}^{j}\right|<2^{-M}$, the point $\mathbf{p}_{i^{\prime}}^{j^{\prime}}$ lies in the right part of the gray wedge drawn in Figure 10, if $t_{i^{\prime}}^{j^{\prime}}>t_{i}^{j}$ and in the left one if $t_{i^{\prime}}^{j^{\prime}}<t_{i}^{j}$. Indeed, using (90), the turning angle of each generated polygon over interval $\left[t_{i}^{j}, t_{i^{\prime}}^{j^{\prime}}\right]$ is less than $\pi$ and according to (86) its length is bigger than $2 r\left|t_{i^{\prime}}^{j^{\prime}}-t_{i}^{j}\right|$. Hence, the point $\mathbf{p}_{i^{\prime}}^{j^{\prime}}$ must be different from $\mathbf{p}_{i}^{j}$. This holds uniformly, i.e., $h_{0}$ is independent of $i^{\prime}, j^{\prime}$.

Clearly, the angle $\frac{\pi}{4}$ of the wedges can be made arbitrarily small. Using this fact, the proof for non-dyadic $t$ comes from continuity of $\mathbf{p}^{h}(t)$ and convexity of the interior of the left and right wedges.

According to this corollary, the refinement rule generates a sequence of points which is locally "well-behaved": it does not contain any loops locally.

Lemma 11 The sequence of vector functions

$$
\left\{\overrightarrow{\mathbf{n}}^{j}(t)\right\}_{j=0}^{\infty}
$$

converges to a continuous vector function $\overrightarrow{\mathbf{n}}(t)$ along the curve $\mathbf{p}(t)$.

Proof: Applying (64) and (66), we get the result.

\subsection{Existence of tangents}

Now, we shall prove the existence of a tangent at each generated point in $P^{\infty}$. We consider sequences of points in $P^{\infty}$. We use the local coordinate system described in Section 2, see Figure 1. Such a coordinate system exists, iff the considered points are distinct. Corollary 10 proves the existence of such a local coordinate system in a certain neighborhood of a generated point. 
Lemma 12 If the conditions (51) and (52) hold, the system of sequences (41) has the following property: Let $\left\{\mathbf{q}_{k}\right\}_{k=0}^{\infty}$ be a convergent sequence of points $\mathbf{q}_{k}=\mathbf{p}_{i_{k}}^{j_{k}} \in \bar{P}^{\infty}$ and

$$
\lim _{k \rightarrow \infty} \mathbf{q}_{k}=\mathbf{q}
$$

where $\mathbf{q}=\mathbf{p}_{I}^{J}$, for some $J \in \mathbb{Z}_{+}$and $I \in\left\{0, \ldots, n_{J}\right\}$. Then

$$
\lim _{j \rightarrow \infty} \alpha\left(\mathbf{q}_{k}, \mathbf{q}, \overrightarrow{\mathbf{n}}_{\mathbf{q}_{k}}, \overrightarrow{\mathbf{n}}_{\mathbf{q}}\right)=\frac{\pi}{2}
$$

and

$$
\lim _{j \rightarrow \infty} \beta\left(\mathbf{q}_{k}, \mathbf{q}, \overrightarrow{\mathbf{n}}_{\mathbf{q}_{k}}, \overrightarrow{\mathbf{n}}_{\mathbf{q}}\right)=0 .
$$

Proof: We can assume without loss of generality that $\mathbf{q}=\mathbf{p}_{0}^{0}$. Further, let $\left\{t_{k}\right\}_{k=0}^{\infty}$ be the sequence of the dyadic parameters corresponding to $\left\{\mathbf{q}_{k}\right\}$. We assume that $t_{k} \neq 0$ for all $k$. Due to Corollary 10 the limit curve $\mathbf{p}(t)$ is locally loopless. Hence, we have $t_{k} \rightarrow 0$.

First, we prove the second part for special sequences. Let $\mathbf{q}_{k}=\mathbf{p}_{i_{k}}^{k}$ be such that $i_{k+1}=2 i_{k}$ or $i_{k+1}=2 i_{k}+1$ for $k=0, \ldots$. Then $\alpha\left(\mathbf{q}_{k}, \mathbf{q}, \overrightarrow{\mathbf{n}}_{\mathbf{q}_{k}}, \overrightarrow{\mathbf{n}}_{\mathbf{q}}\right)=\alpha_{i_{k}}^{k}$ for $k=0,1, \ldots$ Using (58), we get (107) by induction. Similarly, $\beta\left(\mathbf{q}_{k}, \mathbf{q}, \overrightarrow{\mathbf{n}}_{\mathbf{q}_{k}}, \overrightarrow{\mathbf{n}}_{\mathbf{q}}\right)=\beta_{i_{k}}^{k}$ and using (64), we get (108) for this special case.

Now, let the sequence $\left\{\mathbf{q}_{k}\right\}_{k=0}^{\infty}$ of points be arbitrary. Let $t_{k}=\frac{i_{k}}{2^{m_{k}}}$. Then, using (51) and (65), we have

$$
0 \geq \alpha\left(\mathbf{q}_{k}, \mathbf{q}, \overrightarrow{\mathbf{n}}_{\mathbf{q}_{k}}, \overrightarrow{\mathbf{n}}_{\mathbf{q}}\right)-\frac{\pi}{2}=\sum_{l=0}^{l_{k}}\left(\alpha_{l}^{i_{k}}-\frac{\pi}{2}\right) \geq \alpha_{0}^{M_{k}}-\frac{\pi}{2}
$$

for some $l_{k} \in\left\{0, \ldots, n_{k}-2\right\}$ and

$$
M_{k}=\max \left\{m \in \mathbb{Z}_{+} ; 2^{-m}>t_{k}\right\} .
$$

Since $t_{k} \rightarrow 0$ for $M_{k} \rightarrow \infty$, using (107) for the special case proved above, we get the convergence in the general case.

In order to prove (108) in the general case, we have via (52) and (66)

$$
0 \leq \beta\left(\mathbf{q}_{k}, \mathbf{q}, \overrightarrow{\mathbf{n}}_{\mathbf{q}_{k}}, \overrightarrow{\mathbf{n}}_{\mathbf{q}}\right)=\sum_{l=0}^{l_{k}} \beta_{l}^{i_{k}} \leq \beta_{0}^{M_{k}}
$$

Using (108) for the special case, we get the result for all sequences.

The last step is the proof of $G^{1}$ continuity of the limit curve. Since we know the curve is $C^{0}$ according to the Lemma 6 , we prove the existence of the tangent line for every generated point. The normal of this line will be the corresponding generated normal vector at the point.

Theorem 13 If the conditions (51) and (52) hold, then the curve $\mathbf{p}(t)$ is a locally injective curve with a well defined tangent and is therefore $G^{1}$ everywhere.

Proof: Let $\left\{t_{k}\right\}_{k=0}^{\infty}$ be a convergent sequence of dyadic parameters with dyadic limit $t$, and let $\mathbf{q}_{k}=\mathbf{p}\left(t_{k}\right), \mathbf{q}=\mathbf{p}\left(t_{k}\right)$ be the corresponding points. We assume that $t_{k} \neq t$ for all $k$. Then, $\left\{\mathbf{q}_{k}\right\}_{k=0}^{\infty}$ is a convergent sequence of points in $\bar{P}^{\infty}$ and $\mathbf{q}_{k} \rightarrow \mathbf{q} \in \bar{P}^{\infty}$. Moreover, $\mathbf{q}_{k} \neq \mathbf{q}$ for all $k$, due to Corollary 10. Let the generated normal at $\mathbf{q}_{k}$ be $\overrightarrow{\mathbf{m}}_{k}$. We prove that the line $\overleftrightarrow{\mathbf{q q}} \mathbf{q}_{k}$ 

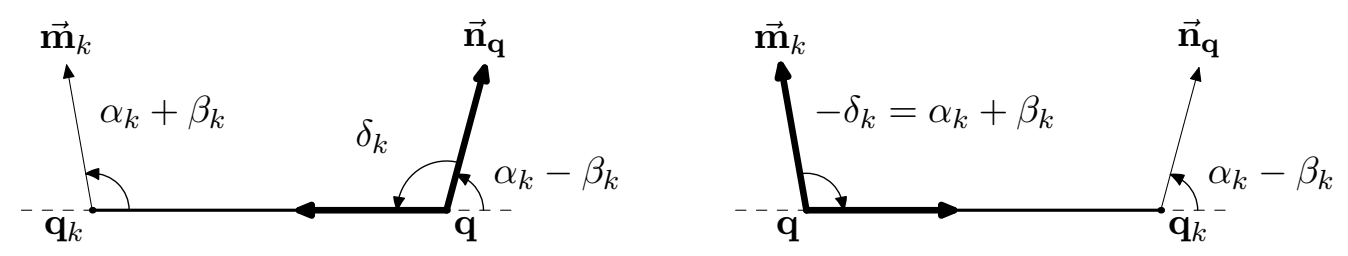

Figure 11: The convergence of the tangent for positive and negative $\delta_{k}$

becomes perpendicular to $\overrightarrow{\mathbf{n}}_{\mathbf{q}}$ in the limit. More precisely, we prove that the angles between the vectors $\overrightarrow{\mathbf{m}}_{k}$ and $\overrightarrow{\mathbf{n}}_{\mathbf{q}}$ converge to 0 ,

$$
\lim _{k \rightarrow \infty} \frac{\mathbf{q}_{k}-\mathbf{q}}{\left\|\mathbf{q}_{k}-\mathbf{q}\right\|} \cdot \overrightarrow{\mathbf{n}}_{\mathbf{q}}=0 .
$$

Since the function $\overrightarrow{\mathbf{n}}(t)$ is continuous according to Corollary 11, we suppose that $\overrightarrow{\mathbf{m}}_{k}$ is in a the neighborhood of $\overrightarrow{\mathbf{n}}_{\mathbf{q}}$. Let $\left\{\alpha_{k}\right\}_{k=0}^{\infty}$ and $\left\{\beta_{k}\right\}_{k=0}^{\infty}$ be the sequences generated by

$$
\alpha_{k}=\alpha\left(\mathbf{q}_{k}, \mathbf{q}, \overrightarrow{\mathbf{m}}_{k}, \overrightarrow{\mathbf{n}}_{\mathbf{q}}\right)
$$

and

$$
\beta_{k}=\beta\left(\mathbf{q}_{k}, \mathbf{q}, \overrightarrow{\mathbf{m}}_{k}, \overrightarrow{\mathbf{n}}_{\mathbf{q}}\right) .
$$

Using Lemma 12 we get (see also Figure 11)

$$
\frac{\mathbf{q}_{k}-\mathbf{q}}{\left\|\mathbf{q}_{k}-\mathbf{q}\right\|} \cdot \overrightarrow{\mathbf{n}}_{\mathbf{q}}=\cos \delta_{k}=\cos \left(\pi-\alpha_{k}+\beta_{k}\right) \rightarrow 0
$$

when $\delta_{k}>0$ and similarly

$$
\frac{\mathbf{q}_{k}-\mathbf{q}}{\left\|\mathbf{q}_{k}-\mathbf{q}\right\|} \cdot \overrightarrow{\mathbf{n}}_{\mathbf{q}}=\cos \delta_{k}=\cos \left(\alpha_{k}+\beta_{k}\right) \rightarrow 0
$$

when $\delta_{k} \leq 0$. This completes the proof for sequences from $P^{\infty}$. Using completion and the fact that $\overrightarrow{\mathbf{n}}(t)$ is $C^{0}$, we conclude that the $\mathbf{p}(t)$ is locally $G^{1}$ with well defined normal $\mathbf{n}(t)$.

\subsection{Examples}

In order to visualize this result, we have checked the quality of the generated curve, as follows. First, we have generated the graph of the normal angle for the generated curve in Figure 3, see Figure 12, (a). Clearly, it is a continuous function of the dyadic parameter.

In Figure 12, (b), the approximation of the curvature (the inverse of the radius of the circle passing through three consecutive generated points) is shown. Both figures are from the level (ix) of the refinement in Figure 3. The limit curve does not seem to have a well-defined curvature.

Since we used circle fits to generate new points, it is clear that this construction reproduces a circle, if the initial data are taken from the circle. This fact is demonstrated by Figure 13, which shows a heart-shaped curve composed of two circular and two non-circular segments.

Finally, we compare the result to standard cubic Hermite splines, where the length of the boundary derivatives was chosen according to the distances $\left\|\mathbf{p}_{i+1}-\mathbf{p}_{i}\right\|$. Clearly, these splines cannot reproduce circles 14(c), while the curve generated by subdivision 14(b) does. 


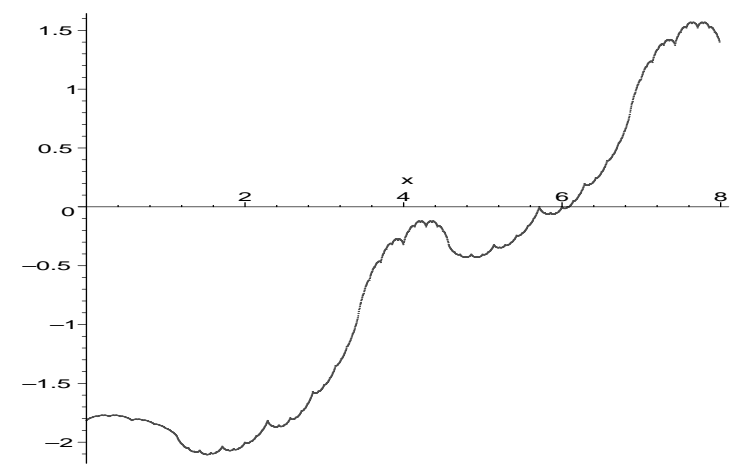

(a)

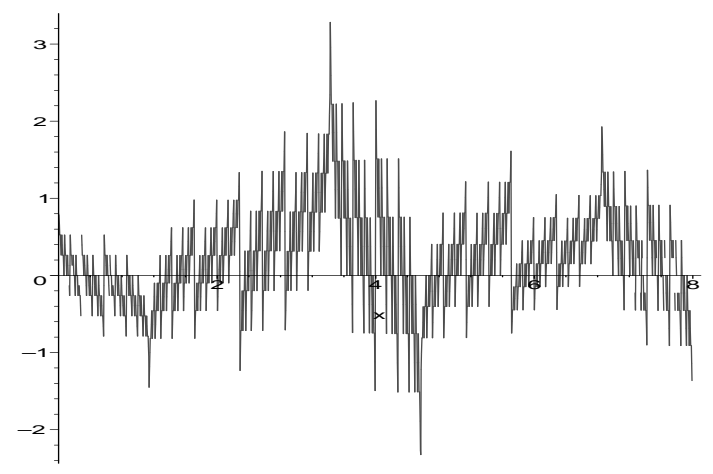

(b)

Figure 12: (a) Graph of the normal angle; (b) Graph of the discrete curvature of the curve
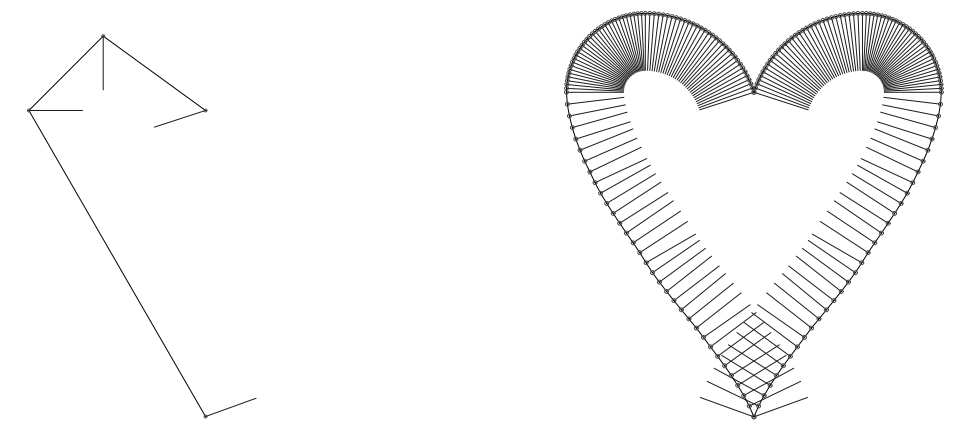

Figure 13: Heart-shaped curve (right) and half of the initial data (left).

\section{Conclusion and Future Work}

We have presented a novel method for refinement of the sequence of points in the plane with associated unit normal vectors. The method is invariant under Euclidean similarities. It produces a $G^{1}$ curve, provided that certain technical conditions are satisfied by the initial data. The initial data (points with associated normals) are interpolated by the generated curve. While the computations are more complicated than for the classical linear subdivision techniques, they are still relatively simple and fairly robust, see Algorithm 2.

Our analysis of the non-linear subdivision relied completely on geometric arguments. More precisely, no special properties of the parameterization (such as asymptotic closeness to a linear subdivision scheme) have been used. As a possible topic for future research, one may try to investigate shape preserving properties of the scheme or a variant thereof. See [6] for more information on shape-preserving interpolatory subdivision.

In order to improve the local behavior of the generated set of points, we have developed an experimental method which generates a smoother normal (see Figure 15). The initial data are taken those from Figure 3, (i). In this case, we fit a general conic section to the set of four consecutive points and their normals. The new point and the corresponding normal are picked from this conic. The construction provides interpolation of the initial points, while the normals are adapted during the refinement. Clearly, this scheme has the property of preserving conics. Following this pattern, subdivision methods that reproduce certain classes 


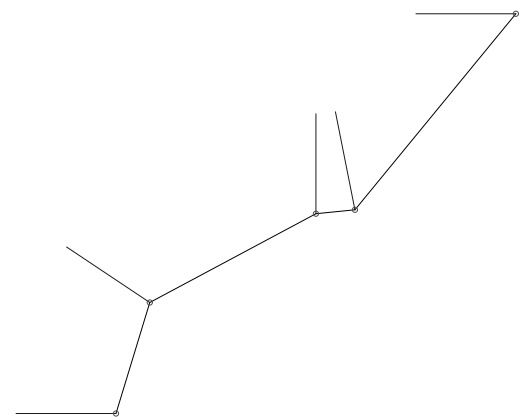

(a)

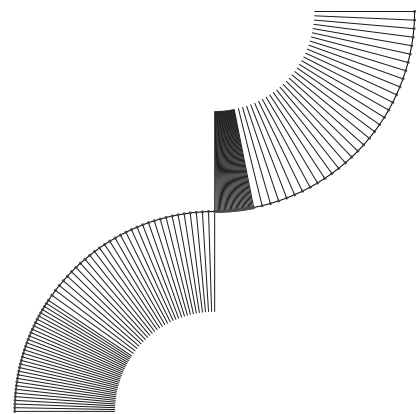

(b)

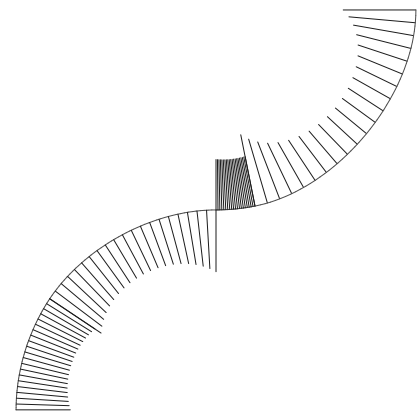

(c)

Figure 14: Comparison of (discrete) curvature of the subdivision curve with cubic Hermite splines. (a) The original data set, (b) subdivision curve, (c) cubic Hermite spline. The scaled normals visualize the curvature distribution.

of curves (e.g., logarithmic spirals) can be developed.

The graph of the normals (see Figure 16, (a)) and the corresponding graph of the curvature radius (see Figure 16, (b)) show a promising behavior. The generated curve and the normal field can be seen on Figure 15. The properties of these curves may be be a subject of further research.

The method can also be extended to the surface case. In this context, we need to consider triangular meshes with given normals in the vertices. The new points can be picked from (e.g.) a quadric fitted to certain neighborhood.

Acknowledgment This research was supported by the Austrian Science Funds (FWF) through the SFB F013 "Numerical and Symbolic Scientific Computing" at Linz, subproject 15. The authors would like to thank the referees for their comments which have helped to improve the manuscript.

\section{References}

[1] N. Aspert, T. Ebrahimi, and P. Vandergheynst. Non-linear subdivision using local spherical coordinates. Comput. Aided Geom. Des., 20(3):165-187, 2003.

[2] N. Dyn and D. Levin. Subdivision schemes in geometric modeling. Acta Numerica, pages 73-144, 2002.

[3] B. Jüttler and U. Schwanecke. Analysis and design of Hermite subdivision schemes. The Visual Computer, 18:326-342, 2002.

[4] S. Karbacher, S. Seeger, and G. Häusler. A non-linear subdivision scheme for triangle meshes. In Bernd Girod et al., editors, Proceedings of the 2000 Conference on Vision Modeling and Visualization, pages 163-170, Saarbrücken, 2000. Aka GmbH.

[5] M.-S. Kim and K.-W. Nam. Interpolating solid orientations with circular blending quaternion curves. Computer-Aided Design, 27(5):385-398, 1995.

[6] F. Kuijt and R. van Damme. Shape preserving interpolatory subdivision schemes for nonuniform data. J. Approx. Theory, 114(1):1-32, 2002.

[7] J.L. Merrien. A family of Hermite interpolants by bisection algorithms. Numer. Alg., pages 187-200, 1992.

[8] G. Morin, J. Warren, and H. Weimer. A subdivision scheme for surfaces of revolution. Comput. Aided Geom. Des., 18(5):483-502, 2001. 

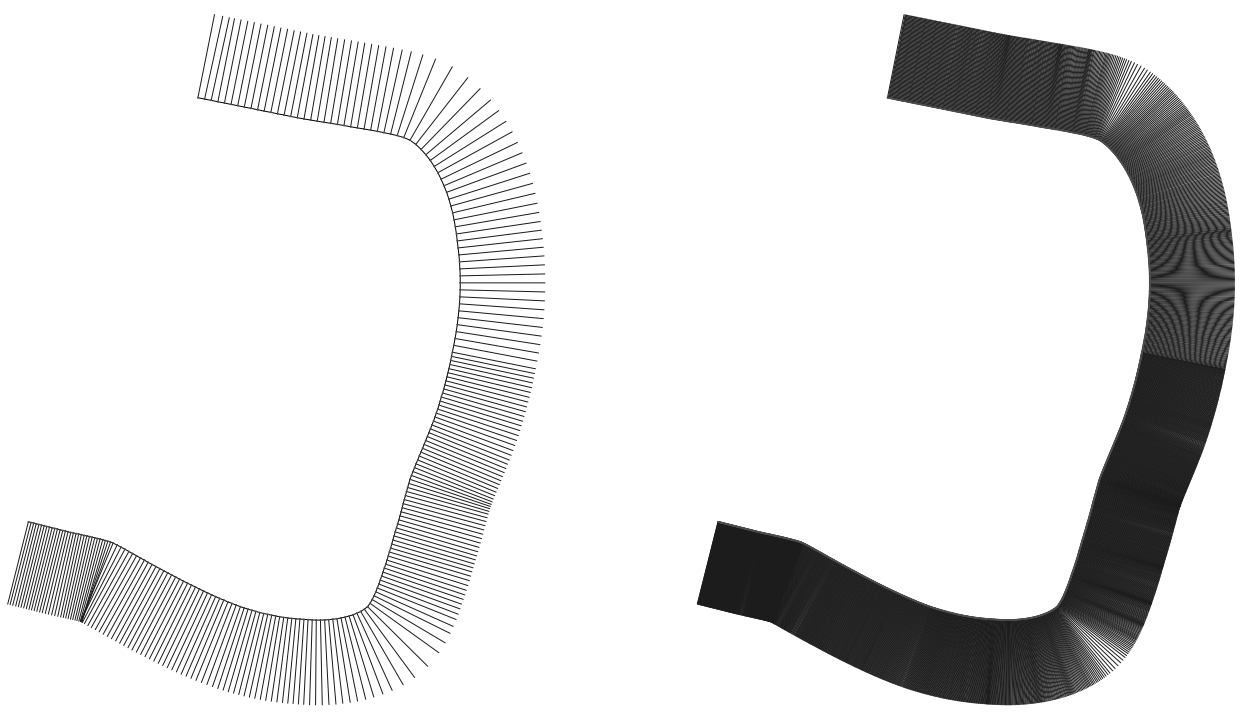

Figure 15: Subdivision via conic fitting. Curves and normals after 5 and 8 subdivision steps.

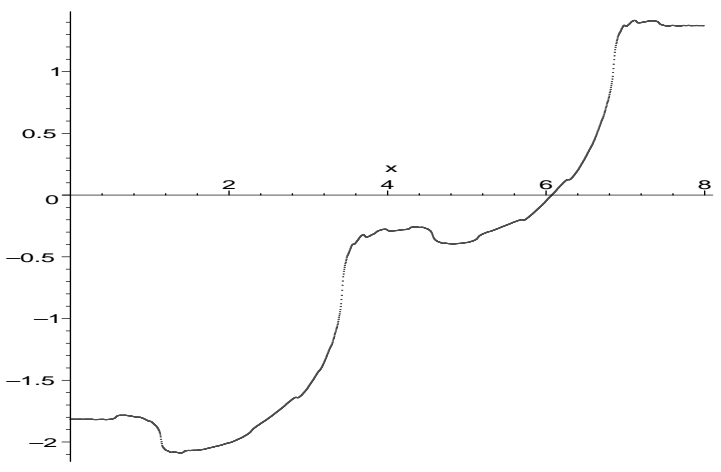

(a)

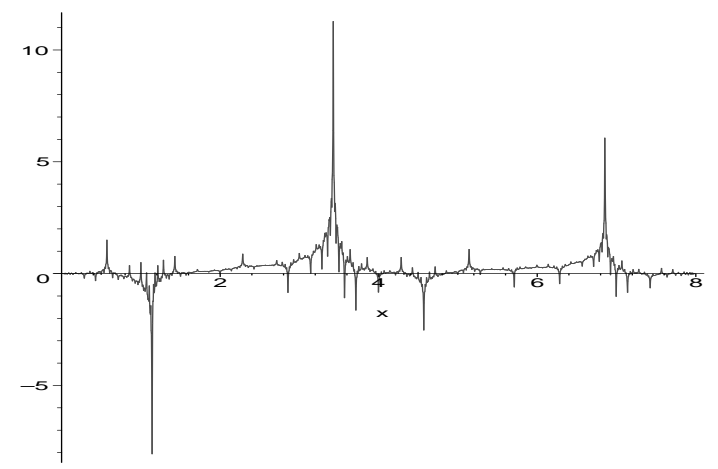

(b)

Figure 16: (a) The graph of the normal angle; (b) Graph of the discrete curvature of the curve in Figure 15.

[9] D.B. Parkinson and D.N. Moreton. Optimal biarc-curve fitting. Computer-Aided Design, 23(6):411-416, 1991.

[10] M. Sabin. A circle-preserving variant of the four-point subdivision scheme. Talk at the Sixth Int. Conference on Mathematical Methods for Curves and Surfaces, Troms $\varnothing 2004$.

[11] C.H. Séquin, K. Lee, and J. Yen. Fair g2 and c2-continuous circle splines for the interpolation of sparse data points. Computer-Aided Design, 37:201-211, 2005.

[12] J. Wallner and N. Dyn. Convergence and $C^{1}$ analysis of subdivision schemes on manifolds by proximity. Comp. Aided Geom. Design, 22, 2005. to appear.

[13] J. Warren. Subdivision Methods For Geometric Design: A Constructive Approach. Morgan Kaufmann, 2003. 